\title{
1 Gut Microbiome Signatures Linked to HIV-1 Reservoir Size and Viremia Control
}

3 Alessandra Borgognone ${ }^{1^{*}}$, Marc Noguera-Julian ${ }^{1,2}$, Bruna Oriol ${ }^{1,3}$, Laura Noël-Romas ${ }^{4,5}$, Marta Ruiz4 Riol $^{1}$, Yolanda Guillén ${ }^{6}$, Mariona Parera ${ }^{1}$, Maria Casadellà ${ }^{1}$, Clara Duran ${ }^{1,3}$, Maria C. Puertas ${ }^{1}$, Francesc

5 Català-Moll ${ }^{1}$, Marlon De Leon ${ }^{4}$, Samantha Knodel ${ }^{4,5}$, Kenzie Birse ${ }^{4,5}$, Christian Manzardo ${ }^{7}$, Jose M. Miró ${ }^{7}$,

6 Bonaventura Clotet ${ }^{1,2,3,8,9}$, Javier Martinez-Picado ${ }^{1,2,10}$, José Moltó ${ }^{8,9}$, Beatriz Mothe ${ }^{1,2,8,9}$, Adam

7 Burgener ${ }^{4,5,11}$, Christian Brander ${ }^{1,2,10}$, Roger Paredes ${ }^{1,2,3,4,8,9^{*}} \&$ the BCN02 Study Group

8

$9 \quad{ }^{1}$ IrsiCaixa AIDS Research Institute, Hospital Universitari Germans Trias i Pujol, Barcelona, Catalonia, Spain

$10 \quad{ }^{2}$ University of Vic - Central University of Catalonia (UVic - UCC), Vic, Catalonia, Spain

$11{ }^{3}$ Universitat Autonoma de Barcelona (UAB), Barcelona, Catalonia,Spain

$12{ }^{4}$ Center for Global Health and Diseases, Department of Pathology, Case Western Reserve University,

13 Cleveland, $\mathrm{OH}$, United States of America

$14{ }^{5}$ Department of Obstetrics \& Gynecology, University of Manitoba, Canada

$15{ }^{6}$ Institut Mar d'Investigacions mediques (IMIM), CIBERONC, Barcelona, Catalonia, Spain

$16{ }^{7}$ Infectious Diseases Service. Hospital Clinic - Institut d'Investigacions Biomèdiques August Pi i Sunyer

17 (IDIBAPS)- University of Barcelona, Barcelona, Catalonia (Spain)

$18{ }^{8}$ Fight AIDS Foundation, Infectious Diseases Department, Germans Trias i Pujol University Hospital,

19 Barcelona, Catalonia, Spain

$20{ }^{9}$ Department of Infectious Diseases Service, Germans Trias i Pujol University Hospital, Barcelona, Catalonia,

21 Spain

$22{ }^{10}$ Catalan Institution for Research and Advanced Studies (ICREA), Barcelona, Catalonia, Spain

$23{ }^{11}$ Department of Medicine Solna, Center for Molecular Medicine, Karolinska Institute, Karolinska University

24 Hospital, Stockholm, Sweden. 


\section{Abstract}

38

\section{$47 \quad$ Results}

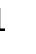

2

3

4

5

6

7

8

69

0

1

\section{Background}

The potential role of the gut microbiome as a predictor of immune-mediated HIV-1 control in the absence of antiretroviral therapy (ART) is still unknown. In the BCN02 clinical trial, which combined the MVA.HIVconsv immunogen with the latency-reversing agent romidepsin in early-ART treated HIV-1 infected individuals, 23\% (3/13) of participants showed sustained low-levels of plasma viremia during 32 weeks of a monitored ART pause (MAP). Here, we present a multi-omics analysis to identify compositional and functional gut microbiome patterns associated with HIV-1 control in the BCN02 trial.

Viremic controllers during the MAP (controllers) exhibited higher Bacteroidales/Clostridiales ratio and lower microbial gene richness before vaccination and throughout the study intervention when compared to noncontrollers. Longitudinal assessment indicated that the gut microbiome of controllers was enriched in proinflammatory bacteria and depleted in butyrate-producing bacteria and methanogenic archaea. Functional profiling also showed that metabolic pathways, including methanogenesis and carbohydrate biosynthesis, were significantly decreased in controllers. Fecal metaproteome analyses confirmed that baseline functional differences were mainly driven by Clostridiales. Participants with high baseline Bacteroidales/Clostridiales ratio had increased pre-existing immune activation-related transcripts. The Bacteroidales/Clostridiales ratio as well as host immune-activation signatures inversely correlated with HIV-1 reservoir size.

\section{Conclusions}

This proof-of-concept study suggests the Bacteroidales/Clostridiales ratio as a novel gut microbiome signature associated with HIV-1 reservoir size and immune-mediated viral control after ART interruption. 
74

\section{Background}

A major obstacle to HIV-1 cure is the persistence of viral reservoirs. This mainly refers to latently-infected cells carrying transcriptionally-silent, replication-competent viruses which evade antiretroviral therapy (ART) as well as immune-mediated clearance[1-3]. The immune system is generally unable to contain HIV-1 replication in the absence of ART[4]. However, up to 10-20\% of subjects that initiate ART within first weeks after HIV-1 acquisition may temporarily achieve HIV-1 viremia suppression after ART interruption (ATI)[5]. Understanding the mechanisms behind immune-mediated viremia control after ATI is key to progress towards a functional HIV cure. Broader and higher-magnitude CTL (cytotoxic T-lymphocyte) responses against less diverse HIV-1 epitopes[6,7] in the context of favorable HLA class I genotypes[8] and smaller HIV-1 reservoir size[9] have all been related to such post-treatment HIV-1 control.

There is indirect evidence that the gut microbiome might also contribute to immune-mediated control of HIV1 replication[10,11]. Vaccine-induced gut microbiome alterations, consisting in lower bacterial diversity and negative correlation between richness and $\mathrm{CD}_{1}{ }^{+} \mathrm{DR}^{-}$monocytes in colorectal intraepithelial lymphocytes, have been recently associated with HIV/SIV (SHIV) protection in a non-human primate challenge study after mucosal vaccination with HIV/SIV peptides, modified vaccinia Ankara-SIV and HIV-gp120-CD4 fusion protein plus adjuvants through the oral route[12]. In the HVTN 096 trial[13], where the impact of the gut microbiota on HIV-specific immune response to a DNA-prime, poxvirus-boost strategy in human adults was assessed, baseline and vaccine-induced gp41-reactive $\operatorname{IgG}$ titers were associated with different microbiota community structures, in terms of richness and composition[14]. In particular, co-occurring bacterial groups, such as Ruminococcaceae, Peptoniphilaceae, and Bacteroidaceae, were associated with vaccine-induced $\operatorname{IgG}$ response and inversely correlated with pre-existing gp41 binding IgG antibodies, suggesting that the microbiome may influence the immune response and vaccine immunogenicity[15]. Further evidence emerged from other studies in typhoid Ty21[16], rotavirus[17] and oral polio virus, tetanus-toxoid, bacillus CalmetteGuérin and hepatitis B immunization strategies[18], in which specific gut microbiome signatures (Bifidobacterium, Streptococcus bovis and Clostridiales, respectively) positively correlated with vaccineinduced immune response. In the absence of immune correlates of viral control, HIV cure trials usually incorporate an ART interruption phase to address the efficacy of a therapeutic intervention[19]. Data on the role of gut microbiome composition in the responsiveness to a curative strategy and the relationship with viral control after ART interruption are lacking. The BCN02 study[20] was a single-arm, proof of concept "kick \& kill" clinical trial evaluating the safety and the in vivo effects of the histone deacetylase inhibitor romidepsin given as a latency reversing agent[21] in combination with a therapeutic HIV vaccine (MVA.HIVconsv) in a group of early-ART treated HIV-1-infected individuals[22,23]. During a monitored ART interruption (MAP), $23 \%$ of individuals showed sustained viremia control up to 32 weeks of follow-up.

Here, we aimed to identify salient compositional and functional gut microbiome patterns associated with control of HIV-1 viremia after ART interruption in the "kick \& kill" strategy used in the BCN02 study. 


\section{Materials and Methods}

112

113

114

115

116

117

118

119

120

121

122

123

124

125

126

127

128

129

130

131

132

133

134

135

136

137

138

139

140

141

142

143

144

145

146

\section{Study design}

This was a sub-study derived from the BCN02 clinical trial (NCT02616874). The BCN02 was a multicenter, open-label, single-arm, phase I, proof of concept clinical trial in which 15 HIV-1-infected individuals with sustained viral suppression who started ART within the first six months after HIV transmission were enrolled to evaluate the safety, tolerability, immunogenicity and effect on the viral reservoir of a kick\&kill strategy consisting of the combination of HIVconsv vaccines with romidepsin[20] (Additional Figures: Figure S1a). Fifteen individuals enrolled in the $\mathrm{BCN} 02$ trial (procedures for recruitment and eligibility criteria are detailed elsewhere[20]) were immunized with a first dose of MVA.HIVconsv (MVA1, $2 \times 10^{8}$ pfu intramuscularly), followed by three weekly-doses of romidepsin $\left(\mathrm{RMD}_{1-2-3}, 5 \mathrm{mg} / \mathrm{m}^{2} \mathrm{BSA}\right.$ intravenously) and a second boost of MVA.HIVconsv (MVA2, $2 \times 10^{8}$ pfu intramuscularly) four weeks after the last $\mathrm{RMD}_{3}$ infusion. To assess the ability for viral control after ART interruption, participants underwent a monitored antiviral pause (MAP), 8 weeks after the second vaccination (MVA2), for a maximum of 32 weeks or until any ART resumption criteria were met (plasma viral load $>2,000$ copies $/ \mathrm{ml}, \mathrm{CD} 4^{+}$cell counts $<500$ cells $/ \mathrm{mm}^{3}$ and/or development of clinical symptoms related to an acute retroviral syndrome[20]). The study was conducted between February 2016 and October 2017 at two HIV-1 units from university hospitals in Barcelona (Hospital Germans Trias i Pujol and Hospital Clínic) and a community center (BCN-Checkpoint, Barcelona). The microbiome sub-study concept, design and patient information were reviewed and approved by the institutional ethical review board of the participating institutions (Reference $\mathrm{Nr}$ AC-15-108-R) and by the Spanish Regulatory Authorities (EudraCT 2015-002300-84). Written informed consent was provided by all study participants in accordance to the principles expressed in the Declaration of Helsinki and local personal data protection law (LOPD 15/1999).

\section{Sample disposition and data analysis}

Fourteen participants from the BCN02 trial consented to participate in the BCN02-microbiome study, one was excluded due to a protocol violation during MAP and thirteen were included for multi-omics analyses. Twelve from the thirteen participants that finalized the "kick \& kill" intervention completed the MAP phase $(\mathrm{n}=3$ controllers and $n=9$ non-controllers) and one subject (B07) did not enter the MAP period due to immune futility pre-defined criteria and absence of protective HLA class I protective alleles associated with natural HIV-1 control (Additional Figures: Figure S1b). Based on the gut microbiome similarity with non-controllers at study entry and over the "kick \& kill" intervention, the participant B07 was included in the non-controller arm to increase the statistical power in this microbiome sub-study.

Fecal specimens were longitudinally collected at BCN02 during the intervention period at study entry (preVax), 1 week after $1^{\text {st }}$ vaccination (MVA1), 1 week after $\mathrm{RMD}_{3}(\mathrm{RMD})$ and 4 weeks after $2^{\text {nd }}$ vaccination (MVA2). Samples were also collected over the MAP period (from 4 to 34 weeks after ART interruption) and 
24 weeks after ART resumption (Additional Figures: Figure S1a). All samples were processed for shotgun metagenomics analysis. Taxonomical classification, microbial gene content and functional profiling were inferred using Metaphlan2[24], IGC reference catalog[25] and HUMAnN2[26], respectively. Sequencing analysis and quality control of metagenomics data are provided in the Additional Results section (Additional Text). To facilitate the interpretation, longitudinal time points were schematically grouped into three phases (Additional Figures: Figure S2a). Fecal material, peripheral blood mononuclear cells (PBMC) and plasma samples were also sampled at baseline to assess fecal metaproteome, host transcriptome profiles and soluble inflammation biomarkers, respectively (Additional Figures: Figure S2b). Microbial proteins from fecal samples were measured by mass spectrometry and protein identification performed using Mascot search engine (v2.4, Matrix Science) and Scaffold Q+ software (v4.9.0, Proteome Software)[27]. PBMC transcriptomes were evaluated using RNA-sequencing and sequence reads aligned to the human reference genome by STAR v2.5.3a[28]. Read counts estimation was inferred using RSEM v1.3.0[29] and differential expression analysis performed by DESeq2[30]. Plasma proteins were estimated using the Proximity Extension Assay based on the Olink Inflammation Panel[31]. Correlations between 'omic' datasets were computed using Spearman's correlation coefficients and integrative multi-omics analysis was assessed based on the mixOmics $\mathrm{R}$ package[32]. A detailed description of wet-lab procedures, bioinformatic methods and statistical analysis of metagenome, metaproteome, transcriptome, soluble plasma markers and multi-omics data is available in the Additional Methods section (Additional Text).

\section{Results}

\section{Patient characteristics}

In this microbiome sub-study, we evaluated 13 participants of the BCN02 study. Three had sustained plasma HIV-1 viremia ( $<2,000$ copies/ml) during 32 weeks of MAP (viremic controllers), whereas 9 developed HIV1 RNA rebound (>2,000 copies/ml) during MAP (non-controllers). One additional subject (B07) did not qualify for MAP due to pre-specified immune futility criteria and absence of protective HLA alleles, and therefore, was also considered a non-controller in this microbiome study. (Additional Figures: Figure S1b). Study participants were predominantly MSM (92\%) of Caucasian ethnicity (92\%), with median age of 42 years and median body mass index of $22.9 \mathrm{~kg} / \mathrm{m}^{2}$ (Table 1). Median baseline CD4 ${ }^{+}$T-cell counts were 728 (416-1408) cells/ $\mathrm{mm}^{3}$ and median CD4/CD8 T-cell ratio was 1.4 (0.97-1.9). All subjects had been on integrase strand-transfer inhibitor-based triple ART for $>3$ years, begun during the first 3 months after HIV-1 infection. Median baseline HIV-1 proviral DNA was 140 copies $/ 10^{6} \mathrm{CD}^{+} \mathrm{T}$-cells, being numerically lower in controllers than in non-controllers ( 65 vs 165 copies $/ 10^{6} \mathrm{CD}^{+} \mathrm{T}$-cells, $p=0.29$ ).

\section{Baseline gut-associated Bacteroidales / Clostridiales ratio discriminates between viremic controllers and} non-controllers 
Viremic controllers had significantly higher Bacteroidales levels than non-controllers at study entry (pre-Vax) ( $p=0.007$ ) and during all the intervention phase (after the first MVA dose: MVA1 $p=0.049$, after the three romidepsin doses: RMD $p=0.049$ and after the second MVA dose: MVA2, $p=0.014$ ) (Fig. 1a, Additional Figures: Figures S3-S4) as well as lower Clostridiales $(p=0.014)$ levels before vaccination (Fig. 1b, Additional Figures: Figures S3-S4). The Bacteroidales/Clostridiales ratio remained significantly higher in controllers throughout the intervention (pre-Vax, $p=0.007$ and MVA2, $p=0.028$ ) (Fig. 1c). In addition, noncontrollers were enriched in Erysipelotrichales and Coriobacteriales (Additional Figures: Figure S4a) and showed significantly higher Methanobacteriales levels (Additional Figures: Figure S4b). More detailed analyses at lower taxonomic levels using the LEfSe algorithm (Additional Figures: Figure S5) showed that controllers were mainly enriched in Prevotella copri, as well as in Haemophilus pittmaniae and Streptococcus parasanguinis. In comparison, non-controllers were enriched in the Clostridiales species Eubacterium rectale and siraeum, Subdoligranulum spp. Coprococcus, and Dorea longicatena, as well as in Collinsella aerofaciens and Methanobrevibacter spp. A longitudinal analysis using the 'feature-volatility' function from qiime2 (Additional Figures: Figure S6) showed that such differences were sustained over the whole intervention period. All 9 non-controllers analyzed here resumed ART by week 4 after the MAP initiation, whereas the 3 controllers remained off ART for at least 28 weeks and up to 32 weeks. During the MAP, Bacteroidales showed an initial increase up to week 4 followed by a reduction by weeks 8-12 in controllers (Fig. 1a). Inversely, Clostridiales levels increased by weeks 8-12 and remained stable thereafter (Fig. 1b) (no statistical support provided during MAP). No significant differences in bacterial composition were found following ART resumption; however, there was a limited sample availability at ART resumption phase (Figs. 1a-c).

\section{Viremic controllers display lower gut microbial diversity and richness over the intervention}

Controllers had lower microbial gene counts than non-controllers at the study entry and throughout the study intervention, although such differences lost statistical significance in the RMD and MVA2 assessments (Fig. 2a). Intra-individual diversity (Shannon index) also remained numerically lower in controllers, but differences were not statistically significant (Fig. 2b). During the MAP, gut microbial diversity increased around weeks 810 in controllers, and remained stable thereafter (Figs. 2a-b). No statistically significant differences were found in microbial diversity following ART re-initiation (Figs. 2a-b). Using the Bray-Curtis index, controllers exhibited lower beta-diversity and higher similarity, particularly already at the study entry (Additional Figures: Figure S7), and showed less intra-host longitudinal evolution (Fig. 2c) than non-controllers (PERMANOVA, $r^{2}=0.591, p=0.001$ ). Whereas the gut microbiome composition of controllers was significantly different from that of non-controllers (PERMANOVA, $r^{2}=0.112, p=0.001$ ), no significant longitudinal differences were observed within each group (PERMANOVA, $r^{2}=0.043, p=0.815$ ), suggesting that the combined intervention with MVA.HIVconsv vaccines and three weekly low-dose infusion of romidepsin did not significantly alter the gut-microbiome composition (Fig. 2c). Of note, results did not change after removing B07 from the non-controller arm (Additional Figures: Figure S8). 


\section{Longitudinal microbial metabolic pathways differ between viremic controllers and non-controllers}

222 Functional profiling based on HUMAnN2[26] identified 28 differential metabolic pathways between

223 controllers and non-controllers at study entry (unadjusted $p<0.05$, Wilcoxon test) (Additional Figures: Figure

224 S9 and S10a). Twelve out of the 28 pathways identified at study entry were differentially abundant throughout

225 the intervention (Additional Figures: Figure S10b). Controllers were enriched in 'fatty acid and lipid biosynthesis' and 'amino acid biosynthesis' pathways. Conversely, metabolic pathways overrepresented in non-controllers included 'methanogenesis from $\mathrm{H}_{2}$ and $\mathrm{CO}_{2}$ ', 'carbohydrate biosynthesis' and 'generation of precursor metabolite and energy'. Longitudinal variations of such metabolic pathways during MAP and after ART re-initiation are shown (Additional Figures: Figure S11), although low numbers did not allow for statistical testing. The 'methanogenesis from $\mathrm{H}_{2}$ and $\mathrm{CO}_{2}$ ' pathway was the most discriminant feature between the two groups (fold-change $=11.5, p=0.04$ ). Consistently, methanogenic archaea (Methanobrevibacter smithii and Methanosphaera stadtmanae) were detected in most non-controllers and were rare or absent in controllers (Additional Figures: Figure S12). Taken together, these data show that differences between controllers and non-controllers emerged from resident microbial communities, before any intervention was started in BCN02 study. Thus, subsequent analyses were focused on characterizing further potentially discriminant signatures at study entry.

Increased Bacteroidales/Clostridiales ratio in viremic controllers negatively correlated with longitudinal HIV-1 viral reservoir size

The Bacteroidales/Clostridiales ratio inversely and significantly correlated with longitudinal total $\mathrm{CD}^{+} \mathrm{T}$ cell-associated HIV-1 DNA measured at study entry (rho $\square=-0.6, p \square=\square 0.03$ ) and over the "kick \& kill" intervention, whereas an opposite trend was observed for gene richness (rho $=0.65, p \square=\square 0.01$ at study entry) (Fig. 3a). Alpha-diversity (Shannon index) exhibited weak positive correlation with the viral reservoir, being the correlation not significant. In line with the gut microbiota patterns found in controllers, the ratio Bacteroidales/Clostridiales showed a strong negative correlation with gene richness $(\mathrm{r} \square \mathrm{ho}=$ 0.87, $p \square=\square 0.0001$ ) (Fig. 3a). In the longitudinal comparison, controllers tended to displayed lower viral reservoir size, although differences were not statistically significant (Fig. 3b). A similar trend was observed for cell-associated (CA) HIV-1 RNA (Figs. 3c-d), although stronger correlations were found at RMD and MVA2 timepoints with both Bacteroidales/Clostridiales ratio (RMD; rho $=-0.76, p=0.002$ and MVA2; rho= $0.74, p=0.003$ ) and gene richness (RMD; rho $=0.72, p=0.005$ and MVA2; rho=0.71, $p=0.006$ ) (Fig. $3 \mathrm{c}$ ).

251 Moreover, in the longitudinal comparison, controllers displayed significantly lower CA HIV-1 RNA at RMD and MVA2 ( $p=0.03$ ) (Fig. 3d). A set of clinical and vaccine-response variables was screened for association with gut microbial signatures. Absolute $\mathrm{CD} 4{ }^{+} \mathrm{T}$-cell count before ART initiation was the only factor significantly associated with the Bacteroidales/Clostridiales ratio (rho $=0.65, p \square=\square 0.01$ ) and gene richness

255 ( $\mathrm{r} \square \mathrm{ho}=-0.62, p \square=\square 0.02$ ), whereas a strong and inverse correlations was found between the Shannon index and CD4/CD8 ratio at BCN02 study entry (rho $\square=0.9, p \square=\square 2.83 \mathrm{e}-05$ ) (Additional Figures: Figure S13). 


\section{Distinct bacterial protein signatures associated with viremia control}

259 Baseline metaproteome analysis identified 15,214 bacterial proteins, annotated to 24 unique orders and 69

260 genera across samples. The abundance of total Clostridiales or Bacteroidales was not different between

261 groups (Figs. 4a-c). However, several Clostridiales genera were decreased in controllers, i.e.: Eubacterium (-

$2623.71 \% ; p=0.03)$, Pseudoflavonifractor (-0.49\%; $p=0.049)$, Oscillibacter $(-0.14 \% ; p=0.07)$, whereas Blautia

263 was increased (+5.02\%; $p=0.03$ ) (Fig. 4a). At the genus level, the relative abundance of Erysipelotrichales (-

$2640.28 \% ; p=0.07)$, and Coprobacillus $(-0.22 \% ; p=0.07)$ showed a decreasing trend in controllers, although

265 differences were not statistically significant (Fig. 4b). Unbiased hierarchical clustering showed protein

266 differences $(p<0.025)$ between groups (Fig. 4d). Viremic controllers were enriched in bacterial proteins from

267 Blautia and Ruminococcus, and depleted in proteins derived from other Clostridiales such as Clostridium,

268 Eubacterium, Coprococcus, Faecalibacterium, Oscillibacter, and Pseudoflavinofactor. Pathways associated

269 with Blautia included galactose, starch/sucrose and glyoxylate/dicarboxylate metabolism as well as ribosome

270 activity. (Fig. 4e). Butyrate and other short-chain fatty acid metabolism pathways were similar in both groups

271 (Figs. 4d-e).

272 Increased baseline immune activation and inflammatory response gene expression in viremic 273 controllers

274 Full-PBMC gene expression analysis detected a total of 27,426 transcripts at baseline, after filtering for lowexpressed genes. Using DESeq2[30], a total of 31 differentially expressed genes (DEGs) were identified $\left(\log _{2}\right.$ FoldChange $=0$ and BH-adjusted $p$-value $<0.1$ ), of which 15 and 16 were upregulated in controllers and noncontrollers, respectively (Fig. 5a). The proportion of protein-coding genes, pseudogenes, non-coding transcripts, TEC (genes to be experimentally confirmed), and long-intergenic noncoding genes within DEGs was $61.29 \%, 12.90 \%, 12.90 \%, 9.68$ and 3.23\%, respectively (Additional Table 1). We found 10 and 3 DEGs showing $\log _{2} \mathrm{FC}$ above 2 and 4 , respectively; moreover 8 out 10 genes with $\log _{2} \mathrm{FC}>2$ at a BenjaminiHochberg-adjusted $p$-value significance $<0.05$ (Fig. 5a and Additional Table 1). Hierarchical clustering based on transcriptional DEG profiles showed that controllers grouped together, while non-controllers separated into two distinct expression groups (Additional Fig. 13a). Upregulated genes in non-controllers included 11 transcripts with unknow function (Additional Table 2), which were excluded from downstream analyses. The most statistically differentially-expressed gene was myeloperoxidase $(M P O$, adjusted $p$-value $=1.38 \mathrm{e}-06)$, followed by a member of the folate receptor family $(F O L R 3$, adjusted $p$-value $=2.68 \mathrm{e}-05)$ (Additional Figs. 13b-c and Additional Table 2). Interestingly, both neutrophil-related transcripts MPO and FOLR3 were upregulated in controllers (Fig. 5b) and have been implicated in immune response signaling and regulation of inflammatory processes[33,34]. Other transcripts with known associations to host defense and neutrophilmediated immunity and higher expression in controllers included defensin alpha 1 and 4 (DEFA1, DEFA4), bactericidal permeability-increasing protein $(B P I)$, cathepsin $\mathrm{G}(C T S G)$ and neutrophil elastase (ELANE) (Fig.

292 5b and Additional Table 2). Gene Ontology (GO) enrichment analysis identified a number of biological 
the complete list of GO terms was collapsed into representative subclasses using REVIGO. Many of these functions were associated with immune response, such as neutrophil-mediate immunity, leukocyte degranulation and antimicrobial humoral response (Fig. 5c).

\section{Increased baseline inflammation-related plasma proteins in viremic controllers}

Soluble factors in plasma from the 92 inflammation-related protein panel were assessed using the Proximity Extension Assay (see Methods in Additional Text). Plasma protein levels did not independently separate controllers from non-controllers using unbiased hierarchical clustering or principal component analysis (Additional Figs. 14a-b). Of the 92 plasma proteins characterized, only 7 were differentially expressed (Wilcoxon, uncorrected $p<0.05$ ) and were increased in controllers (Additional Fig. 14c): adenosine deaminase ADA ( $\mathrm{p}=0.012)$, decoy receptor osteoprotegerin OPG $(\mathrm{p}=0.024)$, self-ligand receptor of the signaling lymphocytic activation molecule family SLAMF-1 $(\mathrm{p}=0.048)$, chemokines CCL23, CCL28, MCP-2 ( $\mathrm{p}=$ $0.048)$ and the neurotrophic factor NT-3 ( $\mathrm{p}=0.048)$ (Additional Fig. 14d).

\section{Integration analysis between Bacteroidales/Clostridiales ratio, host immune activation-related} transcripts, bacterial proteins and HIV-1 reservoir size

The Bacteroidales / Clostridiales ratio was positively correlated (Spearman rho $=0.55$ and $q$-value $<0.05$ ) with DEGs involved in inflammatory response and immune system activation, including DEFA1, DEFA4, TOP1MT, CTSG, MPO, AZU1, ELANE (Fig. 6a), Spearman rho and $q$ value are given in Additional Dataset 1. Functional enrichment analysis including all transcripts significantly correlated with the ratio Bacteroidales/Clostridiales (n. transcripts $=453, q$-value $<0.05$, see Additional Dataset 1 ) identified four main functional clusters related to neutrophil activation, disruption of cells of other organism, antimicrobial humoral response and regulation of immune response to tumor cell (Additional Fig. 15a and Additional Table 4). Of these, the Bacteroidales / Clostridiales ratio strongly correlated (rho >0.8) with transcripts within such functional clusters, which were highly expressed in controllers (Fig. 6b).

Moreover, 61 and 70 out 453 transcripts (Additional Dataset 2) correlated (rho $=0.5$ ) with the baseline CA HIV-1 RNA and HIV-1 DNA, respectively, and were enriched in immune-mediated response functions (Additional Figs. 15b-c and Additional Table 5). In the integrated analysis of metagenomic, transcriptomic and metaproteomic data to identify signatures discriminating between controllers and non-controllers, Bacteroidales and Clostridiales were clearly separated through the component (Additional Fig. 16a). While Bacteroidales clustered with immune activation-related transcripts (MPO, AZU1, ELANE, TCN1, DEFA1, BPI, DEF4) and proteins from Ruminococcus, Blautia, Prevotella and Faecalibacterium genera, Clostridiales inversely correlated with these features (Additional Figs. 16a-b). Such associations were assessed at a lower taxonomic scale and confirmed that Bacteroidales species (B. dorei and B. eggerthii) inversely correlated with HIV-1 DNA levels, whereas members of Clostridiales (S. unclassified, D. formicigenerans and E. siraeum) positively correlated with HIV-1 DNA and CA HIV-1 RNA levels. The viral reservoir (HIV-1 DNA and CA HIV-1 RNA) negatively correlated with genes involved in 'neutrophil mediated immunity', 'antimicrobial 
331 humoral response' and 'cell killing'. Weak correlations were observed with bacterial proteins involved in the

332 regulation of metabolism (Fig. 6c and Additional Dataset 2). Overall, these findings further supported positive

333 associations between Bacteroidales abundance and transcripts related to immune response in controllers,

334 which in turn negatively correlated with the viral reservoir size.

\section{Discussion}

337 In this proof-of-concept study, a longitudinal multi-'omics' analysis identified the Bacteroidales/Clostridiales 338 ratio as a novel gut microbiome signature associated with HIV-1 reservoir size and viremic control during a 339 monitored ART pause. Individuals with high Bacteroidales/Clostridiales ratio showed gene expression 340 signatures related to immune activation, particularly neutrophil-mediated immunity and antimicrobial humoral 341 response, which negatively correlated with the viral reservoir size. Our findings largely arise from 342 unsupervised analyses where many other signatures could have emerged, especially given the relatively low number of individuals analyzed. However, they are internally coherent and consistent with a theoretical framework where increased inflammation might contribute to immune-mediated HIV-1 control. They also 345 suggest a putative biomarker for safer ART interruptions in HIV cure studies.

346 The baseline gut microbiome of controllers was enriched in pro-inflammatory species, such as $P$. copri[35], 347 and depleted in bacteria, traditionally associated with the maintenance of gut homeostasis through production of SCFAs[36], including $R$. intestinalis and Subdoligranulum spp. Lower microbial diversity and gene richness in controllers were consistent with a previous work from our group in people living with HIV[37], as well as other studies[38], in which higher gene richness associated with increased levels of butyrate-producing bacteria and methanogenic archaea. Microbial functional enrichment in 'lipid and fatty acid biosynthesis' in controllers might be reflective of mechanisms of lipopolysaccharide biosynthesis and production of inflammatory mediators[39] mediated by members of Bacteroidales[40]. No discernible longitudinal variations were observed in the gut microbiome of BCN02 participants, in line with a previous evidence in oral typhoid immunization strategy[16]. Of note, the gut microbiome in healthy population has been described generally resilient to perturbations[41]. Taken together, these observations would suggest a trend toward the maintenance of a relative stability in the gut microbiome composition, with resident microbial communities potentially associated with viral control during ART interruption. Baseline metaproteome analysis confirmed that functional differences between controllers and non-controllers were mainly driven by Clostridiales, which were actively producing microbial proteins in both groups albeit in distinct functional contexts. Further discriminating baseline signatures linked to increased immune system activation and inflammatory response in controllers emerged from PBMC transcriptome and inflammation-related plasma proteins profiling. It also emerged that the ratio Bacteroidales/Clostridiales inversely correlated with the viral reservoir size in terms of viral reservoir on ART interruption outcomes[9]. 
367 Taken together, these findings suggest that baseline immune activation potentially associated with a microbial 368 shift toward pro-inflammatory bacteria and lower viral reservoir may contribute to sustained post ART 369 interruption HIV-1 control. While there is evidence suggesting a strong impact of the gut microbiota 370 composition on host immune system and inflammatory status[42], the mechanistic basis of how microbial 371 communities may interact with the viral reservoir and, in turn, exert immunomodulatory effects on HIV-1 372 control during ART interruption remains to be delineated. We speculate that a pre-existing, altered balance of 373 'beneficial' gut microbial groups, such as Clostridiales, and concomitant overabundance of pro-inflammatory 374 bacteria would boost host immune system activation, thus triggering a prompt control of rebounding virus, as 375 observed in controllers. In support of this hypothesis, increased abundance of members from Clostridiales 376 were previously associated with neutrophilia and lower poliovirus and tetanus-hepatitis B vaccine 377 response[18]. Moreover, baseline transcriptional pro-inflammatory and immune activation signatures were 378 suggested as potential predictors of increased influenza[43], systemic lupus erythematosus[44] and hepatitis $\mathrm{B}$ [45] vaccine-induced immune response, with weaker responses in elderly[43,45]. It is thus reasonable to postulate that immune activation prior to vaccination together with microbiome-associated factors may affect 381 vaccine outcomes.

382 This study has several limitations. Due to eligibility criteria in the parental BCN02 study, there was a limited 383 sample size, and we were unable to include a control arm without the intervention. Therefore, our 384 considerations were narrowed to three individuals that showed viremic control during ART interruption. 385 Bearing these limitations in mind, our results should be interpreted with caution, emphasizing the need of independent validation in randomized and placebo-controlled trials to assess potentially unmeasured confounders and provide further perspectives on factors that might induce gut microbial shifts. Upcoming analyses in larger longitudinal trials, including the recently reported AELIX-002 trial[46], where fecal 389 samples have been stored longitudinally, are expected to validate our findings. These preliminary findings might have important implications in the design of HIV-1 cure intervention trials that include ART interruption. As proposed for other therapeutic areas[47], microbiome-associated predictive patterns could help to optimize patient stratification, thus resulting in more targeted studies and higher efficacy of HIV-1 interventions. In addition, if a given resident microbial community is to be defined that is indeed predictive of viral control during ART interruption, then modulating participants' gut microbiota before immunization might potentially modulate vaccine responsiveness and ultimately, clinical outcomes. While host-genetics and other vaccine-associated factors as baseline predictors are less amendable, the gut microbiome is potentially modifiable and even transferrable to another host. Strategies manipulating the gut microbiota composition and relative by-products via prebiotics and/or probiotics administration[48] or microbiota engraftment following fecal microbiota transplantation[49] are under intense evaluation[50], albeit with several limitations. 
402

403

404

405

406

407

408

409

410

411

412

413

414

415

416

417

418

419

420

421

422

423

424

425

426

427

428

429

430

431

432

433

434

435

436

437

438

In conclusion, in this exploratory study, we identified pre-existing gut microbial and immune activation signatures as potential predictors of sustained HIV-1 control in the absence of ART, providing a potential target for future treatment strategies and opening up new avenues for a functional HIV cure.

\section{Availability of data and materials}

Datasets supporting the conclusions of this study are available as Additional information (Additional Datasets). Metagenome and RNA-seq data have been deposited in the European Nucleotide Archive (ENA) and are accessible through ENA accession numbers PRJEB42384 and PRJEB43195. The code and databases used for data analysis are available as Additional information (Additional Code) and at 10.5281/zenodo.4876340.

\section{Abbreviations}

ART: antiretroviral therapy

ATI: antiretroviral therapy interruption

HUMAnN2: HMP unified metabolic analysis network v.2

MAP: monitored antiretroviral therapy pause

MVA: MVA.HIVconsv

RMD: romidepsin

SCFA: short chain fatty acids

\section{References}

1. Volberding PA, Deeks SG. Antiretroviral therapy and management of HIV infection. Lancet. 2010. p. 4962.

2. Finzi D, Hermankova M, Pierson T, Carruth LM, Buck C, Chaisson RE, et al. Identification of a reservoir for HIV-1 in patients on highly active antiretroviral therapy. Science (80- ). 1997;278:1295-300.

3. Siliciano JD, Kajdas J, Finzi D, Quinn TC, Chadwick K, Margolick JB, et al. Long-term follow-up studies confirm the stability of the latent reservoir for HIV-1 in resting CD4+ T cells. Nat Med. 2003;9:727-8.

4. Chun TW, Davey RT, Engel D, Lane HC, Fauci AS. AIDS: Re-emergence of HIV after stopping therapy. Nature. 1999;401:874-5.

5. Namazi G, Fajnzylber JM, Aga E, Bosch RJ, Acosta EP, Sharaf R, et al. The control of HIV after antiretroviral medication pause (CHAMP) study: Posttreatment controllers identified from 14 clinical studies. J Infect Dis. 2018;218:1954-63.

6. Turnbull EL, Wong M, Wang S, Wei X, Jones NA, Conrod KE, et al. Kinetics of Expansion of EpitopeSpecific T Cell Responses during Primary HIV-1 Infection. J Immunol. 2009;182:7131-45.

7. Liu MKP, Hawkins N, Ritchie AJ, Ganusov V V., Whale V, Brackenridge S, et al. Vertical T cell immunodominance and epitope entropy determine HIV-1 escape. J Clin Invest. 2013;123:380-93.

8. Posteraro B, Pastorino R, Di Giannantonio P, Ianuale C, Amore R, Ricciardi W, et al. The link between 

genetic variation and variability in vaccine responses: Systematic review and meta-analyses. Vaccine. 2014;32:1661-9.

9. Li JZ, Etemad B, Ahmed H, Aga E, Bosch RJ, Mellors JW, et al. The size of the expressed HIV reservoir predicts timing of viral rebound after treatment interruption. AIDS. 2016;30:343-53.

10. Zimmermann $P$, Curtis N. The influence of the intestinal microbiome on vaccine responses. Vaccine. 2018. p. 4433-9.

11. Ciabattini A, Olivieri R, Lazzeri E, Medaglini D. Role of the microbiota in the modulation of vaccine immune responses. Front. Microbiol. 2019.

12. Sui Y, Lewis GK, Wang Y, Berckmueller K, Frey B, Dzutsev A, et al. Mucosal vaccine efficacy against intrarectal SHIV is independent of anti-Env antibody response. J Clin Invest. 2019;129:1314-28.

13. Pantaleo G, Janes H, Karuna S, Grant S, Ouedraogo GL, Allen M, et al. Safety and immunogenicity of a multivalent HIV vaccine comprising envelope protein with either DNA or NYVAC vectors (HVTN 096): a phase 1b, double-blind, placebo-controlled trial. Lancet HIV. 2019;6:e737-49.

14. Pantaleo G, Janes H, Tomaras G, Montefiori D, Frahm N, Grant S, et al. Comparing different priming strategies to optimize HIV vaccine antibody responses: results from HVTN 096/EV04 (NCT01799954). AIDS Res Hum retroviruses Conf 2nd HIV Res Prev Conf HIVR4P 2016 United states [Internet]. 2016;32:68. Available from: https://www.cochranelibrary.com/central/doi/10.1002/central/CN-01646903/full

15. Cram JA, Fiore-Gartland AJ, Srinivasan S, Karuna S, Pantaleo G, Tomaras GD, et al. Human gut microbiota is associated with HIV-reactive immunoglobulin at baseline and following HIV vaccination. PLoS One. 2019;14.

16. Eloe-Fadrosh EA, McArthur MA, Seekatz AM, Drabek EF, Rasko DA, Sztein MB, et al. Impact of Oral Typhoid Vaccination on the Human Gut Microbiota and Correlations with S. Typhi-Specific Immunological Responses. PLoS One. 2013;8.

17. Harris VC, Armah G, Fuentes S, Korpela KE, Parashar U, Victor JC, et al. Significant Correlation Between the Infant Gut Microbiome and Rotavirus Vaccine Response in Rural Ghana. J Infect Dis. 2017;215:34-41.

18. Huda MN, Lewis Z, Kalanetra KM, Rashid M, Ahmad SM, Raqib R, et al. Stool microbiota and vaccine responses of infants. Pediatrics. 2014;134.

19. Julg B, Dee L, Ananworanich J, Barouch DH, Bar K, Caskey M, et al. Recommendations for analytical antiretroviral treatment interruptions in HIV research trials-report of a consensus meeting. Lancet HIV. 2019. p. e259-68.

20. Mothe B, Rosás-Umbert M, Coll P, Manzardo C, Puertas MC, Morón-López S, et al. HIVconsv Vaccines and Romidepsin in Early-Treated HIV-1-Infected Individuals: Safety, Immunogenicity and Effect on the Viral Reservoir (Study BCN02). Front Immunol. 2020;11.

21. Wei DG, Chiang V, Fyne E, Balakrishnan M, Barnes T, Graupe M, et al. Histone Deacetylase Inhibitor Romidepsin Induces HIV Expression in CD4 T Cells from Patients on Suppressive Antiretroviral Therapy at Concentrations Achieved by Clinical Dosing. PLoS Pathog. 2014;10. 
476 22. Mothe B, Manzardo C, Sanchez-Bernabeu A, Coll P, Morón-López S, Puertas MC, et al. Therapeutic

477 Vaccination Refocuses T-cell Responses Towards Conserved Regions of HIV-1 in Early Treated Individuals

478 (BCN 01 study). EClinicalMedicine. 2019;11:65-80.

479 23. Létourneau S, Im EJ, Mashishi T, Brereton C, Bridgeman A, Yang H, et al. Design and pre-clinical

480 evaluation of a universal HIV-1 vaccine. PLoS One. 2007;2.

481 24. Truong DT, Franzosa EA, Tickle TL, Scholz M, Weingart G, Pasolli E, et al. MetaPh1An2 for enhanced 482 metagenomic taxonomic profiling. Nat. Methods. 2015. p. 902-3.

483 25. Li J, Wang J, Jia H, Cai X, Zhong H, Feng Q, et al. An integrated catalog of reference genes in the human 484 gut microbiome. Nat Biotechnol. 2014;32:834-41.

485 26. Franzosa EA, McIver LJ, Rahnavard G, Thompson LR, Schirmer M, Weingart G, et al. Species-level 486 functional profiling of metagenomes and metatranscriptomes. Nat Methods. 2018;15:962-8.

487 27. Klatt NR, Cheu R, Birse K, Zevin AS, Perner M, Noël-Romas L, et al. Vaginal bacteria modify HIV 488 tenofovir microbicide efficacy in African women. Science (80- ). 2017;356:938-45.

489 28. Dobin A, Davis CA, Schlesinger F, Drenkow J, Zaleski C, Jha S, et al. STAR: ultrafast universal RNA-seq 490 aligner. Bioinformatics [Internet]. 2013 [cited 2017 Feb 22];29:15-21. Available from: 491 http://www.ncbi.nlm.nih.gov/pubmed/23104886

492 29. Li B, Dewey CN. RSEM: Accurate transcript quantification from RNA-Seq data with or without a 493 reference genome. BMC Bioinformatics. 2011;12.

494 30. Love MI, Huber W, Anders S. Moderated estimation of fold change and dispersion for RNA-seq data with 495 DESeq2. Genome Biol. 2014;15.

496 31. Assarsson E, Lundberg M, Holmquist G, Björkesten J, Thorsen SB, Ekman D, et al. Homogenous 96-plex 497 PEA immunoassay exhibiting high sensitivity, specificity, and excellent scalability. PLoS One. 2014;9.

498 32. Rohart F, Gautier B, Singh A, Lê Cao KA. mixOmics: An R package for 'omics feature selection and 499 multiple data integration. PLoS Comput Biol. 2017;13.

500 33. Aratani Y. Myeloperoxidase: Its role for host defense, inflammation, and neutrophil function. Arch. 501 Biochem. Biophys. 2018. p. 47-52.

502 34. Holm J, Hansen SI. Characterization of soluble folate receptors (folate binding proteins) in humans.

503 Biological roles and clinical potentials in infection and malignancy. Biochim. Biophys. Acta - Proteins 504 Proteomics. 2020.

505 35. Iljazovic A, Roy U, Gálvez EJC, Lesker TR, Zhao B, Gronow A, et al. Perturbation of the gut microbiome 506 by Prevotella spp. enhances host susceptibility to mucosal inflammation. Mucosal Immunol. 2020;

507 36. Lopetuso LR, Scaldaferri F, Petito V, Gasbarrini A. Commensal Clostridia: Leading players in the 508 maintenance of gut homeostasis. Gut Pathog. 2013.

509 37. Guillén Y, Noguera-Julian M, Rivera J, Casadellà M, Zevin AS, Rocafort M, et al. Low nadir CD4+ T510 cell counts predict gut dysbiosis in HIV-1 infection. Mucosal Immunol. 2019;12:232-46.

511 38. Le Chatelier E, Nielsen T, Qin J, Prifti E, Hildebrand F, Falony G, et al. Richness of human gut 512 microbiome correlates with metabolic markers. Nature. 2013;500:541-6. 
513 39. Medzhitov R, Janeway C. J. Advances in immunology: Innate immunity. N Engl J Med. 2000;343:338-

51444.

515 40. d'Hennezel E, Abubucker S, Murphy LO, Cullen TW. Total Lipopolysaccharide from the Human Gut

516 Microbiome Silences Toll-Like Receptor Signaling. mSystems. 2017;2.

517 41. Fassarella M, Blaak EE, Penders J, Nauta A, Smidt H, Zoetendal EG. Gut microbiome stability and

518 resilience: Elucidating the response to perturbations in order to modulate gut health. Gut. 2020.

519 42. Zheng D, Liwinski T, Elinav E. Interaction between microbiota and immunity in health and disease. Cell

520 Res. 2020. p. 492-506.

521 43. Avey S, Cheung F, Fermin D, Frelinger J, Gaujoux R, Gottardo R, et al. Multicohort analysis reveals

522 baseline transcriptional predictors of influenza vaccination responses. Sci Immunol. 2017;2.

523 44. Kotliarov Y, Sparks R, Martins AJ, Mulè MP, Lu Y, Goswami M, et al. Broad immune activation 524 underlies shared set point signatures for vaccine responsiveness in healthy individuals and disease activity in 525 patients with lupus. Nat Med. 2020;26:618-29.

526 45. Fourati S, Cristescu R, Loboda A, Talla A, Filali A, Railkar R, et al. Pre-vaccination inflammation and B527 cell signalling predict age-related hyporesponse to hepatitis B vaccination. Nat Commun. 2016;7.

528 46. Lucia Bailon, Anuska Llano, Samandhy Cedeño, Miriam B. Lopez, Yovaninna Alarcón-Soto, Pep Coll,

529 Àngel Rivero, Anne R. Leselbaum, Ian McGowan, Devi SenGupta, Bonaventura Clotet, Christian Brander,

530 Jose Molt BM. A placebo-controlled ATI trial of HTI vaccines in early treated HIV infection. CROI - Virtual

531 Conf Retroviruses Opportunistic Infect. 2021.

532 47. Boessen R, Heerspink HJL, De Zeeuw D, Grobbee DE, Groenwold RHH, Roes KCB. Improving clinical 533 trial efficiency by biomarker-guided patient selection. Trials. 2014;15.

534 48. Wilson NL, Moneyham LD, Alexandrov AW. A Systematic Review of Probiotics as a Potential 535 Intervention to Restore Gut Health in HIV Infection. J Assoc Nurses AIDS Care. 2013;24:98-111.

536 49. Vujkovic-Cvijin I, Rutishauser RL, Pao M, Hunt PW, Lynch S V., McCune JM, et al. Limited engraftment 537 of donor microbiome via one-time fecal microbial transplantation in treated HIV-infected individuals. Gut 538 Microbes. 2017;8:440-50.

539 50. Rosel-Pech C, Chávez-Torres M, Bekker-Méndez VC, Pinto-Cardoso S. Therapeutic avenues for restoring 540 the gut microbiome in HIV infection. Curr. Opin. Pharmacol. 2020. p. 188-201.

\section{Acknowledgements}

543 The authors thank all volunteers for participating in this study and the BCN02 study group.

BCN02 Study Group. IrsiCaixa AIDS Research Institute-HIV ACAT Hospital Universitari Germans Trias i Pujol, Badalona, Spain: Susana Benet, Christian Brander, Samandhy Cedeño, Bonaventura Clotet, Pep Coll, Anuska Llano, Javier Martinez-Picado, Marta Marszalek, Sara Morón-López, Beatriz Mothe, Roger Paredes,

548 Maria C. Puertas, Miriam Rosás-Umbert, Marta Ruiz-Riol. Fundació Lluita contra la Sida, Infectious Diseases 549 Department, Hospital Universitari Germans Trias i Pujol, Badalona, Spain: Roser Escrig, Silvia Gel, Miriam 
550

551

552

553

554

555

556

557

558

559

560

561

562

563

564

565

566

567

568

569

570

571

572

573

574

575

576

577

578

579

580

581

582

583

584

585

586

López, Cristina Miranda, José Moltó, Jose Muñoz, Nuria Perez-Alvarez, Jordi Puig, Boris Revollo, Jessica Toro. Germans Trias i Pujol Research Institute, Badalona, Spain: Ana María Barriocanal, Cristina PerezReche. Clinical Pharmacology Unit, Hospital Universitari Germans Trias i Pujol, Badalona, Spain: Magí Farré. Pharmacokinetic/pharmacodynamic modeling and simultation, Institut de Recerca de l'Hospital de la Santa Creu i Sant Pau-IIB Sant Pau, Barcelona, Spain: Marta Valle. Hospital Clinic- HIVACAT, IDIBAPS, University of Barcelona, Barcelona, Spain: Christian Manzardo, Juan Ambrosioni, Irene Ruiz, Cristina Rovira, Carmen Hurtado, Carmen Ligero, Emma Fernández, Sonsoles Sánchez-Palomino, and Jose M. Miró. Projecte dels NOMS-Hispanosida, BCN Checkpoint, Barcelona, Spain: Antonio Carrillo, Michael Meulbroek, Ferran Pujol and Jorge Saz. The Jenner Institute, The Nuffield Department of Medicine, University of Oxford, UK: Nicola Borthwick, Alison Crook, Edmund G. Wee and Tomás $\square$ Hanke.

\section{Funding}

This study was funded by Instituto de Salud Carlos III through the project "PI16/01421" (co-funded by European Regional Development Fund "A way to make Europe"). The project was sponsored in part by Grifols and received funding from the European Union's Horizon 2020 Research and Innovation Programme under Grant Agreement $N^{\circ} 847943$ (MISTRAL). The BCN02 clinical trial was an investigator-initiated study funded by the ISCIII PI15/01188 grant, the HIVACAT Catalan research program for an HIV vaccine and the Fundació Gloria Soler. Some sub-analyses of the BCN02 trial were partly funded by the European Union's Horizon 2020 research and innovation program under grant agreement 681137-EAVI2020 and by NIH grant P01-AI131568. A.Bu lab was supported by grants from the Canadian Institutes of Health Research (HB3164066) and the National Institutes for Health Research (R01DK112254). J.M.P lab was supported by grant PID2019-109870RB-I00 from the Spanish Ministry of Science and Innovation and in part by Grifols. J.M.M. received a personal 80:20 research grant from Institut d'Investigacions Biomèdiques August Pi i Sunyer (IDIBAPS), Barcelona, Spain, during 2017-21.

\section{Author Contributions}

R.P, B.M, J.M, B.C, J.M.M and C.B, conceived and designed the study. B.M, R.P, C.M, J.M.M and C.B recruited the study participants and performed their clinical evaluations. M.P and M.C performed fecal DNA extraction, library preparation and sequencing, under the supervision of M.N.J, Y.G and R.P. B.O and C.D performed PBMC transcriptomics and soluble factors determinations, under the supervision of M.R.R and C.B. M.C.P performed the viral reservoir size determinations, under the supervision of J.M.P. L.N.R, M.D.L, S.K and K.B performed fecal metaproteomics experiments and data analysis, under the supervision of A.Bu. A.Bo performed bioinformatics and statistical analyses of metagenome, transcriptome, soluble factors, clinical and integration data, under the supervision of M.N.J and R.P. L.N.R performed the bioinformatics and statistical analyses of fecal metaproteome data, under the supervision of A.Bu. F.C.M, M.N.J, A.Bo, B.O and Y.G contributed to data management. A.Bo and R.P wrote the paper, which was reviewed, edited and approved by all authors. 
bioRxiv preprint doi: https://doi.org/10.1101/2021.10.03.462590; this version posted October 4, 2021. The copyright holder for this preprint (which was not certified by peer review) is the author/funder, who has granted bioRxiv a license to display the preprint in perpetuity. It is made available under aCC-BY-NC-ND 4.0 International license.

587

588 Competing interests

589 The authors declare no competing interests.

590

591

592

593 
a

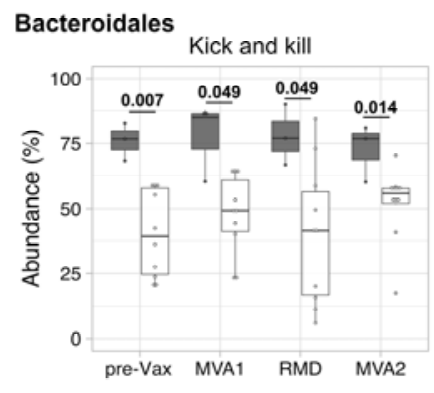

b

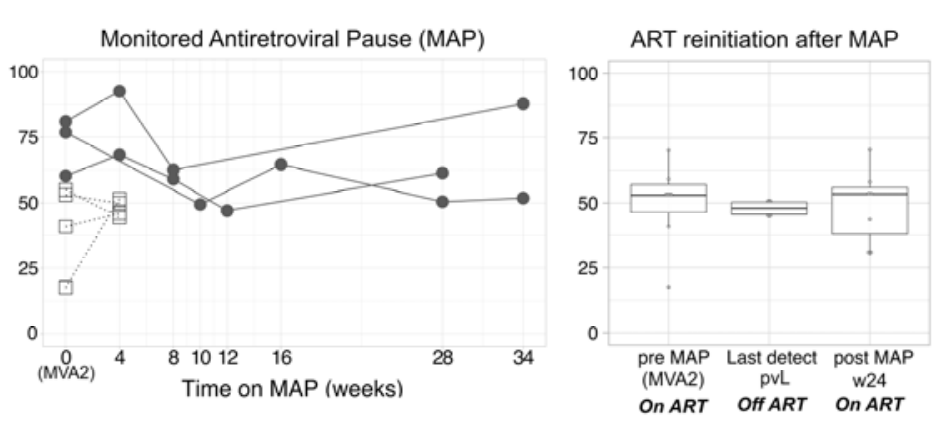

Clostridiales
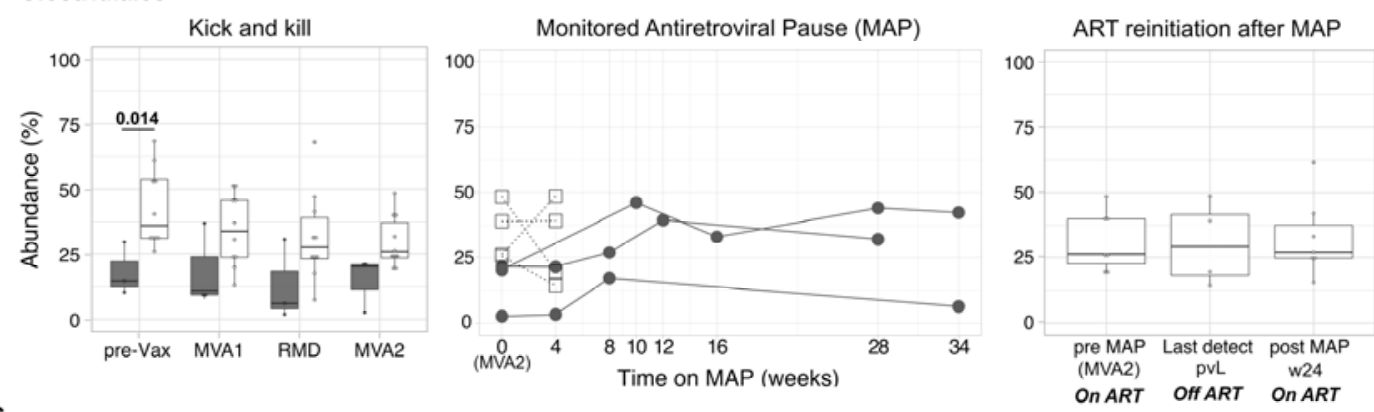

C

Bacteroidales/Clostridiales ratio
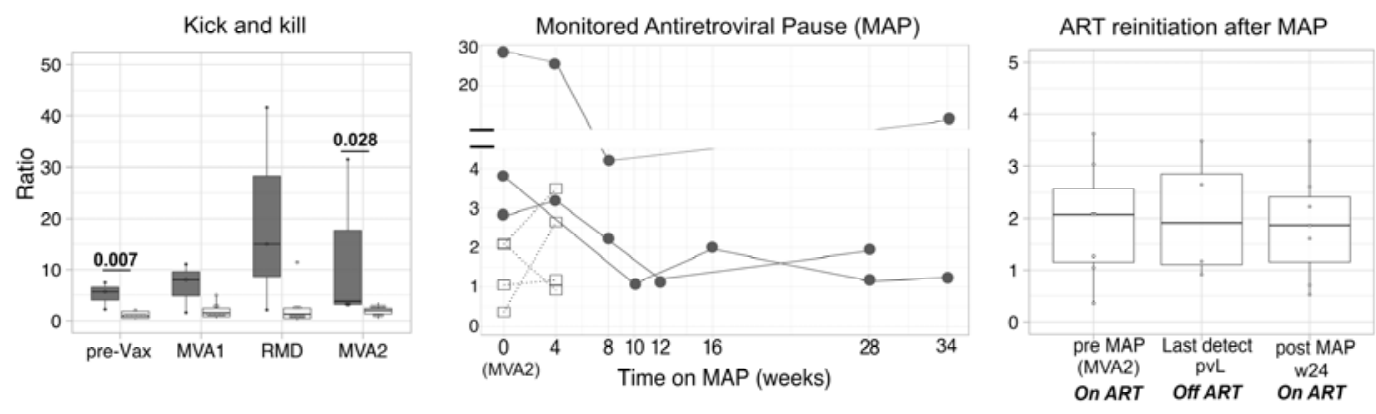

Fig 1. Higher longitudinal Bacteroidales/Clostridiales ratio in viremic controllers. Relative abundance expressed as percentage of (a), Bacteroidales, (b) Clostridiales and (c) their ratio in controllers (gray) and non-controllers (white) are represented by boxplots (left and right vertical panels) and line plots (middle vertical panels). In line plots, values for each subject are illustrated by white squares (non-controllers) and grey dots (viremic controllers). Boxplots show the median (horizontal black line) and interquartile range between the first and third quartiles $\left(25^{\text {th }}\right.$ and $75^{\text {th }}$, respectively). Third vertical panels show non-controllers before ART interruption (pre-MAP, $\mathrm{n}=7$ ), last timepoint on MAP before ART resumption (Last detect pVL, $\mathrm{n}=4$ ) and 24 weeks after ART resumption (post MAP w4, n=7). Abbreviations: MAP, monitored antiretroviral pause; pre-Vax, baseline (1 day before first MVA vaccination); MVA1, 1 week after first MVA vaccination; 

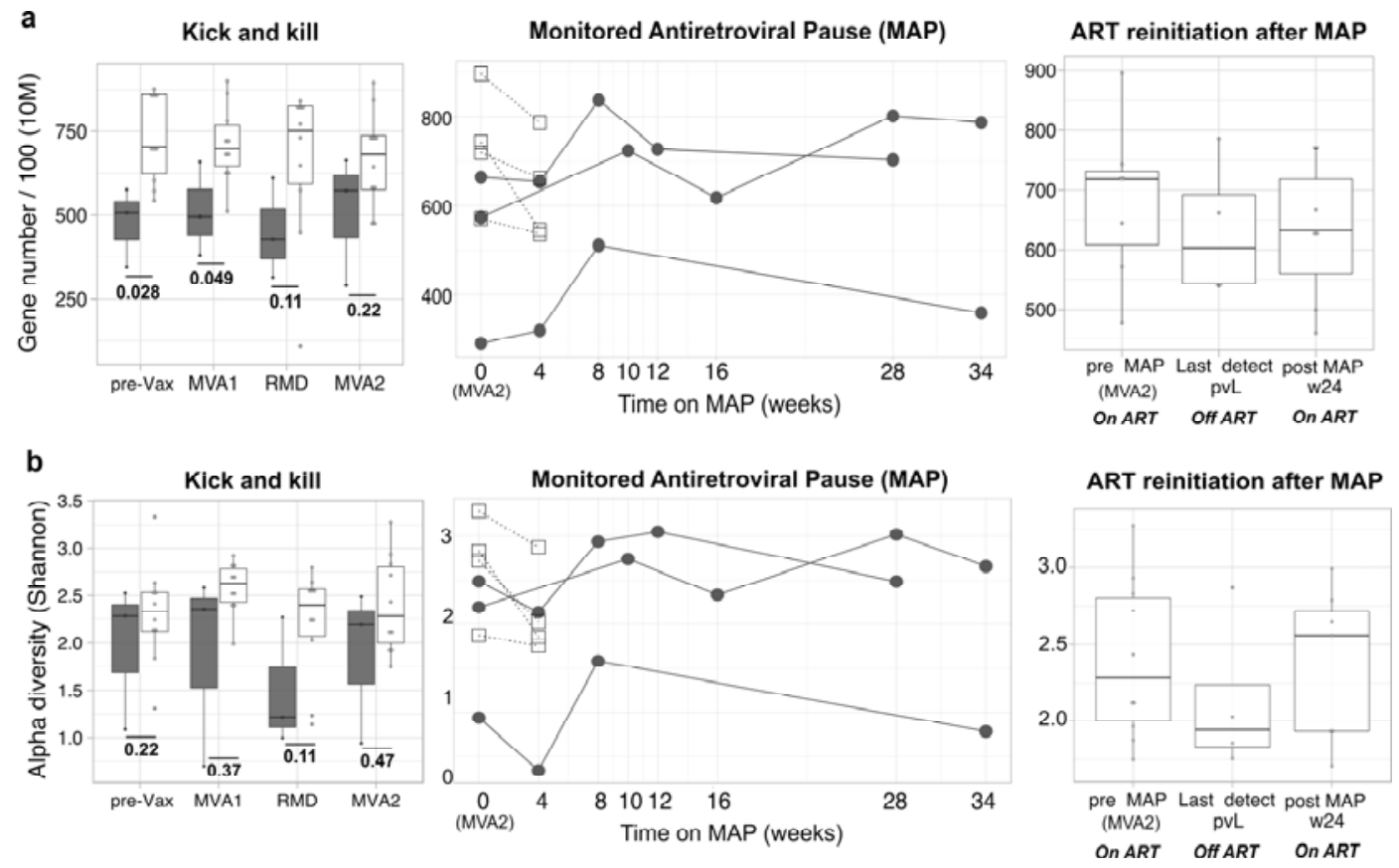

c

- controllers $\boxminus$ non-controllers

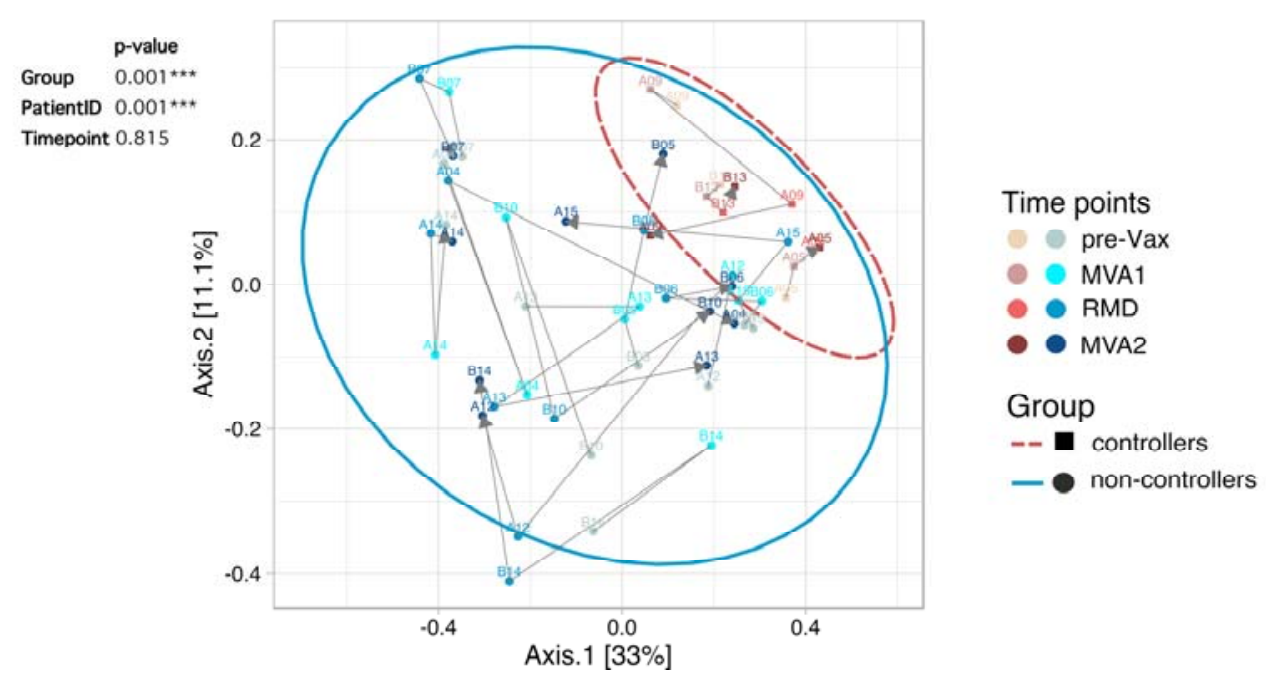

610 Fig 2. Lower microbial diversity and richness in controllers. Longitudinal (a) microbial gene richness at

61110 million (10M filtered reads) down-sampling size and (b) alpha diversity based on Shannon index in

612 viremic controllers (gray) and non-controllers (white). c, Principal coordinates analysis (PCoA) of microbial

613 diversity based on Bray-Curtis distances at pre-vaccination and during 'kick and kill' intervention. Proportion

614 of variance explained by each principal coordinate axis is reported in the corresponding axis label. Subjects

615 per each group are represented by squares (controllers) and circles (non-controllers). Each point stands for one

616 subject, color coded by group and time point. The increase in purple (controllers) and blue (non-controllers)

617 colors reflects sequential time points from baseline (pre-Vax) to the second vaccine administration (MVA2).

618 Ellipses delineate the distribution of points per each group. Gray arrows link directional changes in bacterial abundance throughout the kick and kill intervention from baseline (pre-Vax). PERMANOVA statistical 
620 analysis of samples grouped by Group, PatientID (patient internal identifier) and time point is shown on the

621 top of the panel. Abbreviations: MAP, monitored antiretroviral pause; pre-Vax, baseline (1 day before first

622 MVA vaccination); MVA1, 1 week after first MVA vaccination; RMD, 1 week after third romidepsin

623 infusion; MVA2, 4 weeks after second MVA vaccination.

624

625

626

627

628

629

630

631

632

633

634

635

636

637

638 
a

b

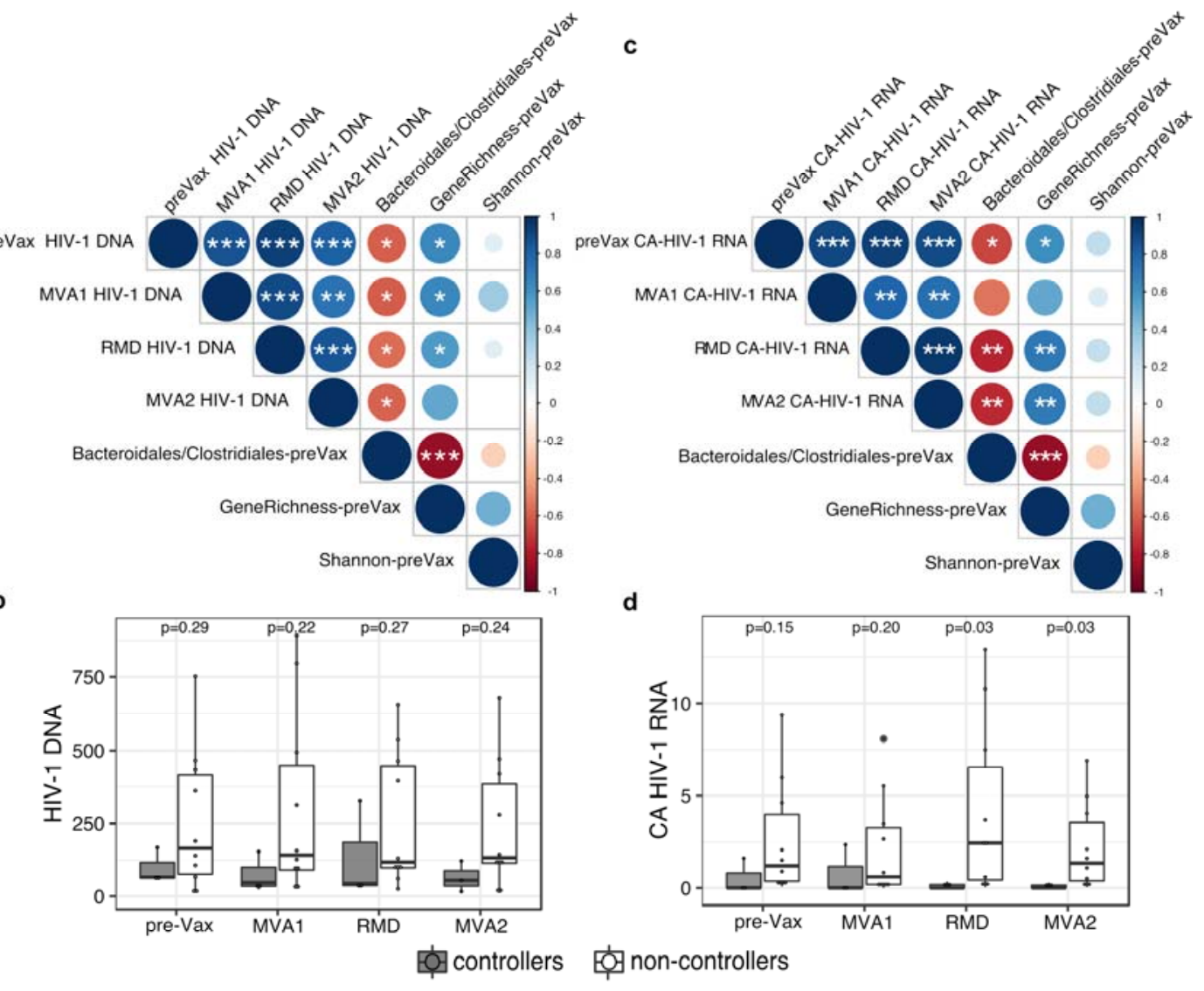

c
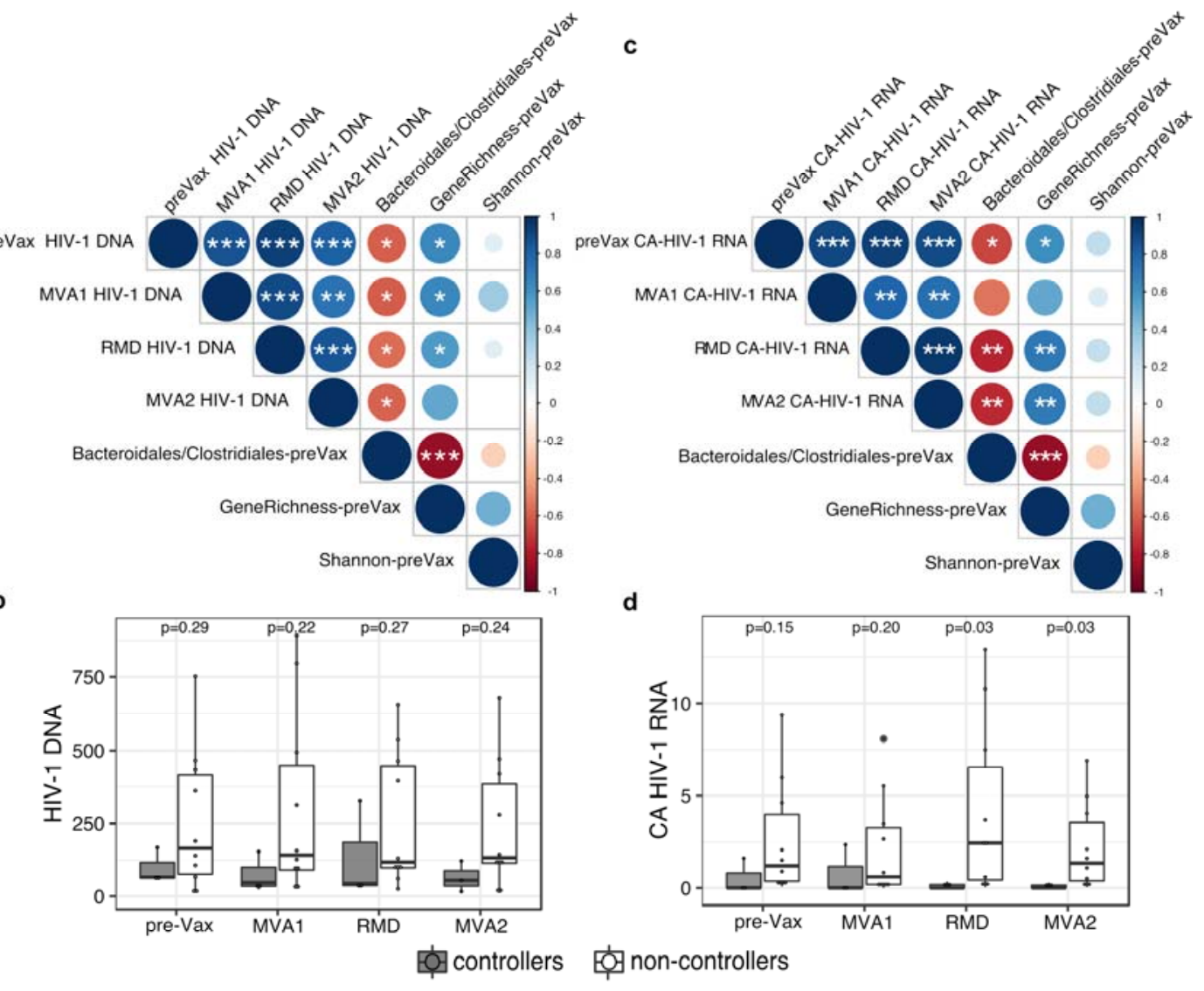

640

641

642

643

644

645

646

647

648

649

650

651

652

653

654

655

Fig 3. Associations between HIV-1 reservoir size and gut microbial signatures. Spearman's correlations between gut microbial signatures (ratio Bacteroidales/Clostridiales, gene richness and alpha-diversity Shannon index) and longitudinal (a) HIV-1 DNA (HIV-1 DNA copies $/ 10^{6}$ CD4 ${ }^{+}$T-cells) and (c) cellassociated (CA) HIV-1 RNA (HIV-1/TBP relative expression). Positive correlations are indicated in blue and negative correlations, in red. Color and size of the circles indicate the magnitude of the correlation. White asterisks indicate significant correlations $\left(* p<0.05 ;{ }^{*} p<0.01 ; * * *<0.001\right.$, Benjamini-Hochberg adjustment for multiple comparisons). Boxplots showing longitudinal comparison of (b) HIV-1 DNA and (d) cell-associated (CA) HIV-1 RNA between controllers and non-controllers. Abbreviations: MAP, monitored antiretroviral pause; pre-Vax, baseline (1 day before first MVA vaccination); MVA1, 1 week after first MVA vaccination; RMD, 1 week after third romidepsin infusion; MVA2, 4 weeks after second MVA vaccination.

d

GeneRichness-preVax

Shannon-preVax

ظonn-controllers

Bacteroidales/Clostridiales-preVax

39



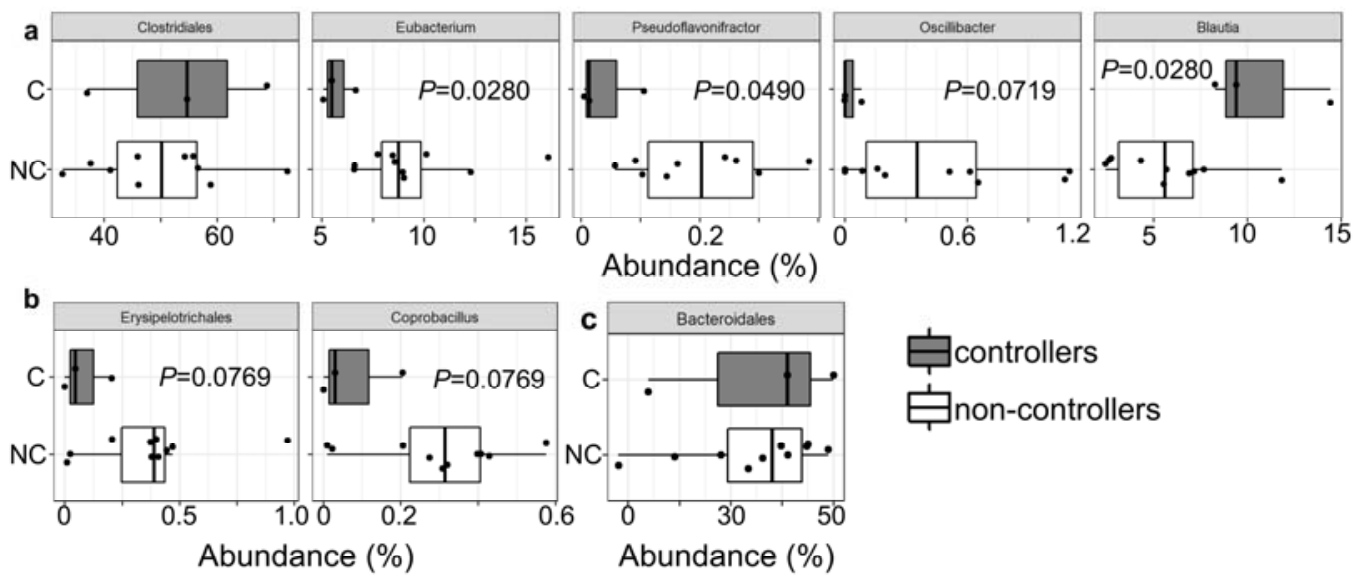

d Clostridiales Proteins
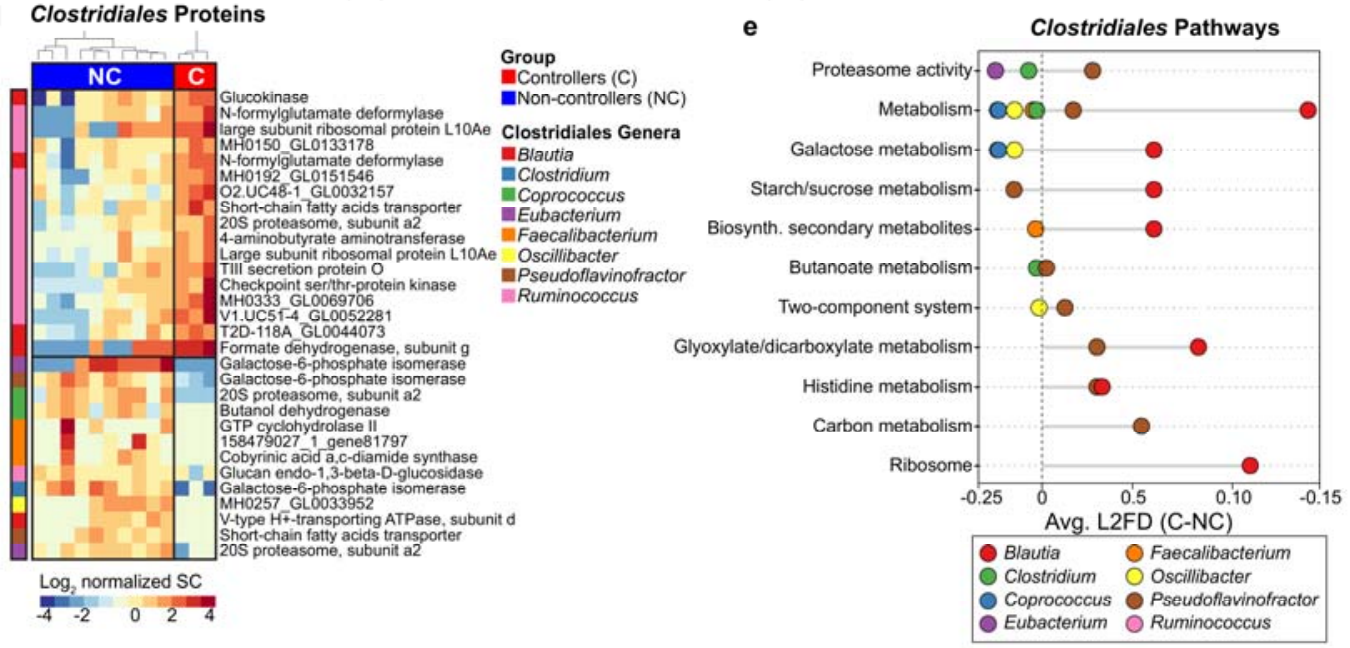

Fig 4. Baseline metaproteomic signatures associated with HIV control after ART interruption. a-c, Metaproteomic analysis of gut microbiome in $\mathrm{BNC} 02$ participants. Proteins from Clostridiales genera and Erysipelorichales were relatively underabundant in controllers compared to non-controllers at baseline prevaccination. No differences in Clostridiales or Bacteroidales proteome at the order-level were observed. d, Baseline levels of Clostridiales proteins (96 proteins) distinguished controllers from non-controllers. Overabundant proteins belonged to the Blautia and Ruminococcus genera, while under-abundant proteins belonged to the Clostridium, Coprococcus, Eubacterium, Faecalibacterium, Oscillibacter and ontology identified differences in cellular metabolism pathways at baseline between groups. Abbreviations:

$667 \mathrm{C}=$ controllers, $\mathrm{NC}=$ non-controllers, $\mathrm{SC}=$ spectral count. 


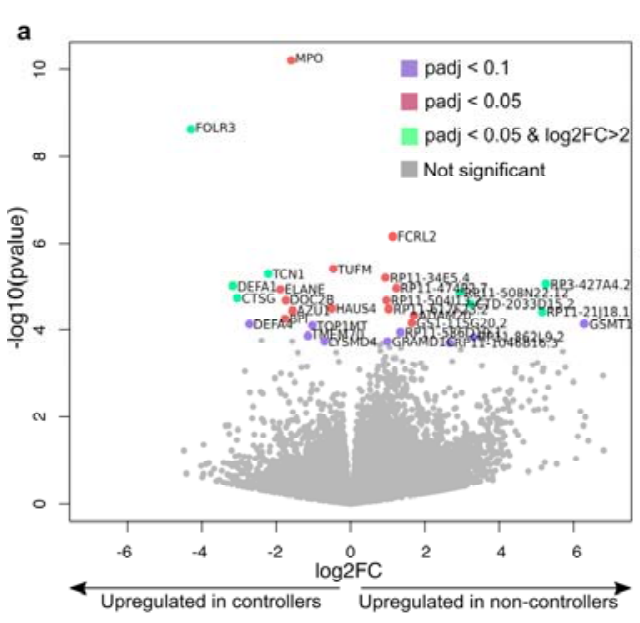

c

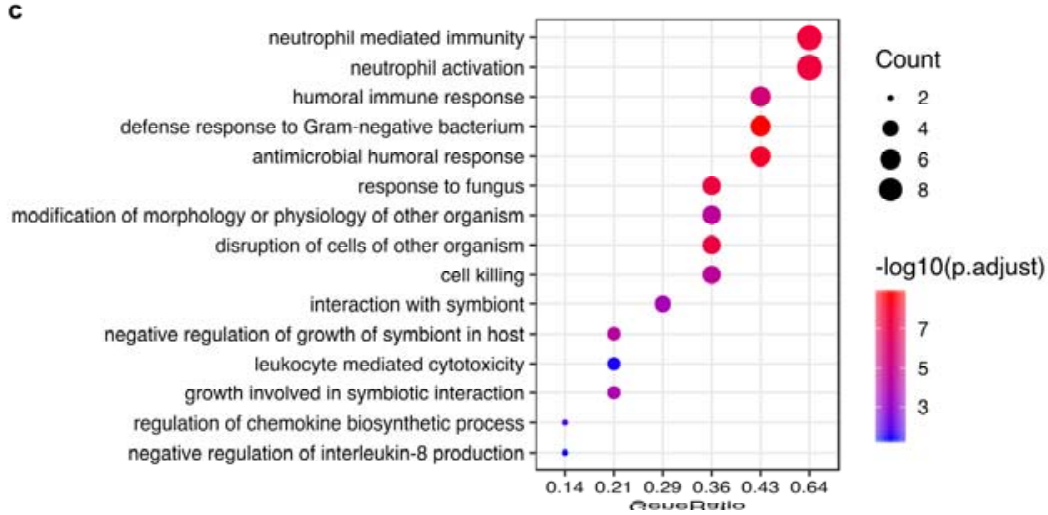

b

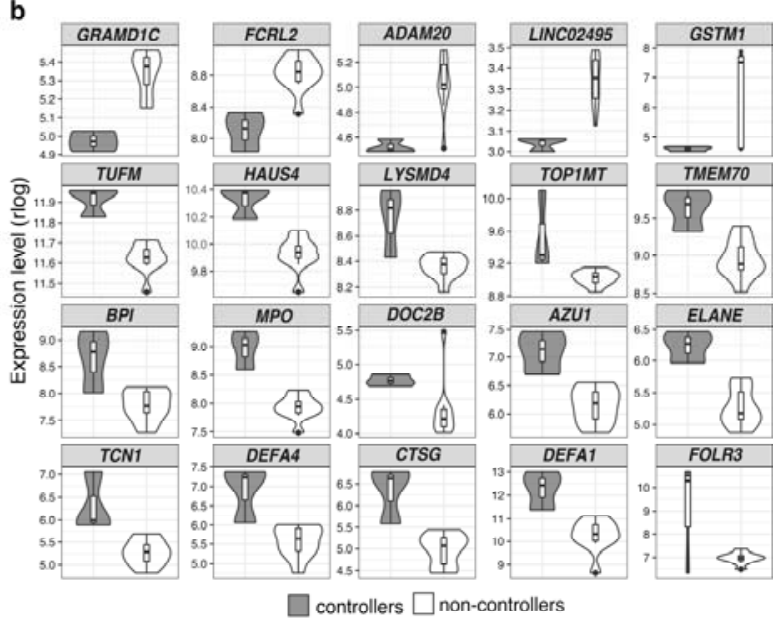

Fig 5. Baseline functional enrichment in levels of immune activation and inflammatory response in viremic controllers. a, Volcano plot of differentially expressed genes between controllers and non-controllers at baseline (pre-Vax) with adjusted $p$-value $<0.1$ (violet dots), adjusted $p$-value $<0.05$ (red dots) and $\log 2$ (FoldChange) $>2$ and adjusted $p$-value $<0.05$ (green dots). Gray-colored dots represent genes not displaying statistical significance (adjusted $p$-value > 0.1). The $\log 2$ Fold Change on the $\mathrm{x}$-axis indicates the magnitude of change, while the $-\log 10$ (p-value) on the y-axis indicates the significance of each gene. A total of 31 genes showed significant differential expression (adjusted $p$-value $<0.1$ ): 15 and 16 upregulated in controllers and non-controllers, respectively. b, Violin plots showing relative expression levels (rlog, regularized log transformation) of differentially expressed genes with functional annotation. c, Gene ontology (GO) enrichment analysis of upregulated genes in Controllers. In the y-axis, only representative enriched GO terms (biological process) are reported (terms obtained after redundancy reduction by REVIGO). X-axis reports the percentage of genes in a given GO terms, expressed as 'Gene ratio'. The color key from blue to red indicates low to high Bonferroni-adjusted log 10 -value. Dot sizes are based on the "count" (genes) associated to each GO term. Significantly enriched GO terms, number of genes associated to each GO term and adjusted $p$-values are provided in Additional Table 3. 
a

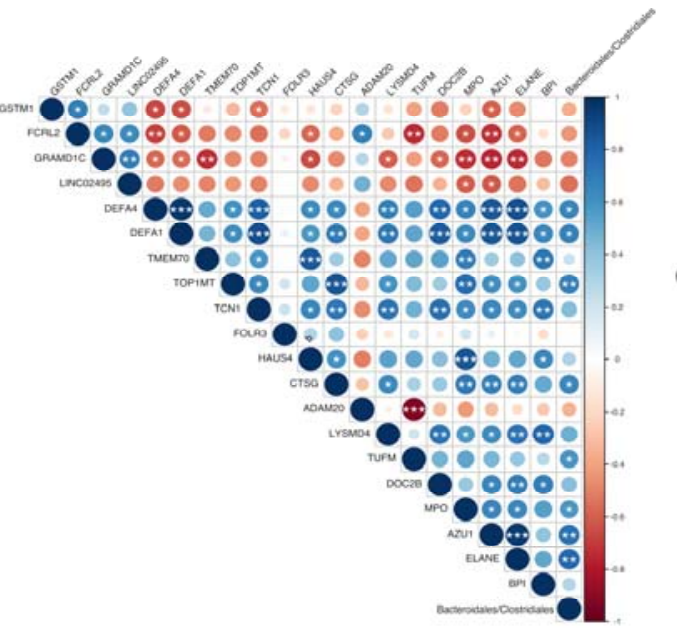

c

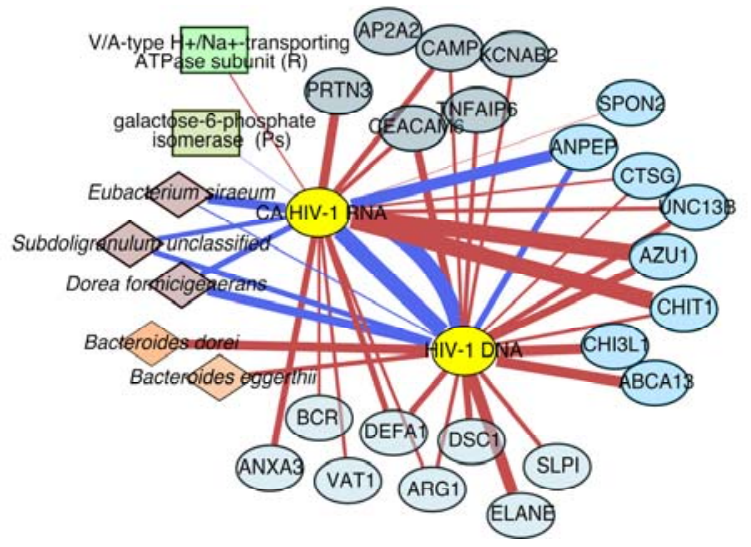

b

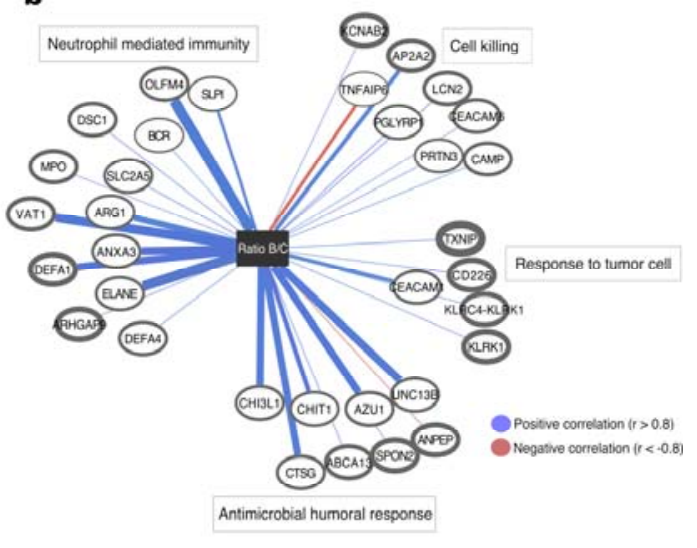

Bacteria

$\checkmark$ Bactroidales

$\checkmark$ Clostridiales

Annotated transcripts

Neutrophil mediated immunity

Antimicrobial humoral response

Cell killing

Microbial proteins

$\square$ Galactose metabolism

$\square$ Metabolism

HIV-1 viral reservoir - preVax

positive correlation $(r>0.5)$

negative correlation $(r<-0.5)$
689

690

691

692

693

694

695

696

697

698

699

700

701

702

703

704

705

Fig 6. Integrative analysis of baseline gut microbial signatures, immune activation-related transcripts, bacterial proteins and HIV-1 reservoir. a, Spearman's correlations between the ratio Bacteroidales/Clostridiales and DEGs (annotated transcripts). Positive correlations are indicated in blue and negative correlations, in red. Color and size of the circles indicate the magnitude of the correlation. White asterisks indicate significant correlations $(* \mathrm{p}<0.05 ; * * \mathrm{p}<$ $0.01 ; * * * p<0.001$, Benjamini-Hochberg adjustment for multiple comparisons). b, Network visualizing significant Spearman's correlations between the ratio Bacteroidales/Clostridiales and transcripts involved in the enrichment analysis described in Additional Table 4. Transcripts are represented as vertices and border width is proportional to transcript expression (log2 |cpmTMM_w0 +1|) in controllers. Edge width indicates the magnitude of correlation. Red and blue edges represent positive and negative correlation, respectively. c, Network showing Spearman's correlation between viral reservoir (CA HIV-1 RNA and HIV-1 DNA), bacterial species within Bacteroidales and Clostrdiales, human transcripts correlated with the ratio Bacteroidales / Clostrdiales and differentially abundant bacterial proteins $(p \leq 0.025)$. Features are showed as vertices and colored by 'omic' dataset. Positive and negative correlations are presented as blue and red edges, respectively. Edge width indicates the magnitude of correlation 
706 coefficient. Protein-associated bacterial genera are reported in parentheses. Abbreviations:

707 DEGs, differentially expressed genes between controllers and non-controllers; R,

708 Ruminococcus; Ps, Pseudoflavonifactor and pre-Vax, baseline timepoint (1 day before first

709 MVA vaccination).

710

711

712

713

714

715

716

717

718

719

720

721

722

723

724

725

726

727

728

729

730

731

732

733

734

735

736

737

738

739

740

741

742 


\section{Tables}

745

Table 1. Study participant demographics and clinical characteristics.

\begin{tabular}{lccc}
\hline Variable & $\begin{array}{c}\text { All participants } \\
(\mathrm{n}=13)\end{array}$ & $\begin{array}{c}\text { non-controllers } \\
(\mathrm{n}=10)\end{array}$ & $\begin{array}{c}\text { controllers } \\
(\mathrm{n}=3)\end{array}$ \\
\hline Demographics & & & $3 / 0$ \\
Sex (M/F), $n$ & $12 / 1$ & $9 / 1$ & $3 / 0$ \\
Risk group (MSM/HTS), $n$ & $12 / 1$ & $9 / 1$ & $3 / 0$ \\
Ethnic group (Caucasian/Latin), $n$ & $12 / 1$ & $9 / 1$ & $34(33-38)$ \\
Age (years) & $42(39-47)$ & $43(39-47)$ & $24.3(22.2-25)$ \\
BMI (Kg/m $\left.{ }^{2}\right)$ & $22.9(20.9-24)$ & $22.3(21.1-23.4)$ & $0 / 3 / 0$ \\
\hline Treatment and clinical characteristics & & $2 / 6 / 2$ & $65(62.5-116.5)$ \\
ART regimen, $n$ & $2 / 9 / 2$ & $165(76.2-415.7)$ & $657(652.5-814)$ \\
(TDF_FTC_RAL/ABC_3TC_RAL/ABC_3TC_DTG) & $140(65-361)$ & $839(581.8-1293.8)$ & $42.2(38.4-48.1)$ \\
Viral reservoir (HIV-1 DNA cp/10 ${ }^{6}$ CD4 ${ }^{+}$T-cells) & $728(648-1182)$ & $43.4(42.3-48.1)$ & $1.3(1.1-1.6)$ \\
CD4 ${ }^{+}$T-cell (cells/mm $\left.{ }^{3}\right)$ & $42.9(42.2-49.3)$ & $1.4(1.2-1.5)$ & \\
CD4 ${ }^{+}$T-cell $(\%)$ & $1.4(1.2-1.6)$ & & \\
CD4/CD8 T-cell counts ratio & & & \\
\hline
\end{tabular}

747 Continuous data are presented using median, $25 \%$ and $75 \%$ interquartile range, unless otherwise 748 described.

749 M, male; F, female; MSM, men who have sex with men; HTS, heterosexual; BMI, body mass 750 index; ART, antiretroviral therapy; cp, copies; TDF, Tenofovir Disoproxil Fumarate; FTC, 751 Emtricitabine; RAL, Raltegravir; ABC, Abacavir; 3TC, Lamivudine; DTG, Dolutegravir. No 752 statistically significant differences were observed $(p \leq 0.05$; Wilcoxon rank-sum test 
bioRxiv preprint doi: https://doi.org/10.1101/2021.10.03.462590; this version posted October 4,2021 . The copyright holder for this preprint (which was not certified by peer review) is the author/funder, who has granted bioRxiv a license to display the preprint in perpetuity. It is made available under aCC-BY-NC-ND 4.0 International license. 